\title{
Wandel der Politikmodi: veränderte Voraussetzungen - neue Formen
}

von Dietrich Fürst

\begin{abstract}
Modi politischer Entscheidungsprozesse auf Bundesebene wandeln sich in Reaktion auf veränderte Rahmenbedingungen (wachsende soziale und materielle Entscheidungskomplexität, Europäisierungs- und Globalisierungsprozesse, Dominanz des Wirtschaftlichen über das Politische etc.). Die Veränderungen richten sich auf so unterschiedliche Bereiche wie eine verstärkte Elitenkooperation, eine Intensivierung von Steuerungsprozessen durch Zielvorgaben/Rahmensetzungen (parametrische Steuerung, vor allem aufgrund wachsender Spielräume im konkreten Vollzug), die Einflussnahme auf Denkmuster und Einstellungen der Akteure (paradigmatische Steuerung) sowie auf eine sich noch weiter intensivierende Auslagerung von politischen Entscheidungen auf vorbereitende Gremien (etwa Kommissionen oder den ubiquitären Einsatz von Beratungsfirmen, nicht zuletzt bei der Formulierung von Gesetzentwürfen). Letztlich geht es im Wesentlichen um die Fähigkeit und Befähigung eines ,, coping “, mithin sich den veränderten Voraussetzungen anzupassen.
\end{abstract}

In Germany, the observable modes of policy-making at the federal level appear to undergo a certain transformation in reaction to changing conditions (growing complexity, Europeanisation and globalisation, dominance of economics over politics, etc.). These changes seem to include more cooperation between the elites involved in political decisions (either as deciding or affected actors); intensified steering by goal-setting/framework-setting, not least due to increasing leeway in questions of implementation; the growing use of learning and teaching processes that seek to influence mind-sets and attitudes; and a further intensification of the ubiquitous transfers of political decision-making to external groups or even private consultants (not least in the preparation of complex legislation). Ultimately, all this boils down to the an enhanced ability to „cope" with the issues at hand.

\section{Einleitung: veränderte Voraussetzungen politischer Entschei- dungsprozesse}

Verfolgt man die laufenden Veränderungen im politischen Prozess Deutschlands und anderer EU-Mitgliedstaaten, so verfestigt sich der Eindruck, dass sich die eingesetzten Politikmodi beträchtlich verändern. Als Politikmodi sollen hier vor allem Verfahren und Prozesse der Konsensbildung und Entscheidung verstanden werden. Was man in diesem Kontext zu beobachten glaubt, ist eine Entwicklung 
hin zu mehr Informalität, zu verstärkten Informationsstrategien, schließlich zu institutionellen Änderungen, um die politischen Kosten immer komplexer werdender Entscheidungsfelder beherrschbar zu halten und die Effektivität politischer Prozesse zu verbessern. Dieser Beitrag versucht, die erkennbaren Veränderungen darzustellen und zu systematisieren.

Unbestritten ist, dass das zeitgenössische Politikmanagement nicht nur komplexer und unübersichtlicher geworden ist, es lässt sich auch immer weniger mit den Routinen politischer Entscheidungsprozesse vereinbaren. Die Gründe hierfür unterliegen vielfältiger Diskussion: ${ }^{1}$

- So werden die Probleme für Entscheider immer komplexer. Auch wenn diese Komplexität objektiv vielleicht nur marginal verändert ist, erweist sie sich für Entscheider subjektiv als gewachsen. Trivial formuliert: ,Auch Politiker, Wissenschaftler und andere Experten ächzen unter den ungeheuren Informationsfluten, der allgemeinen Beschleunigung und den großen Komplexitäten". ${ }^{2}$

- Die zu berücksichtigenden Rahmenbedingungen sind zudem in beträchtlichem Ausmaß ,europäisiert“, in Teilen ,globalisiert“, darüber hinaus internetbasiert und durch einen hohen Grad der Vernetzung gekennzeichnet, wodurch Informationen wiederum schneller transferiert, aber auch „Mitläufer-Effekte" ${ }^{\text {*3 }}$ verstärkt werden.

- Zudem wächst die Zahl derer, die an Entscheidungen mitwirken, deutlich, zum Teil aufgrund der Vielzahl von Mitbestimmungsmöglichkeiten, die den Adressaten von Entscheidungen eröffnet wurden, zum Teil aufgrund weit ausgefächerter Möglichkeiten zur informellen Einflussnahme, etwa über Regionalkonferenzen, Stadtteilforen u.a.m., was die Organisation von Massenaufrufen einschließt; ${ }^{4}$ darüber hinaus greifen veränderte administrative Steuerungsformen, die den nachgeordneten Einrichtungen größere Freiräume im Prozess des Politikvollzugs einräumen. So werden statt der Bindung der beteiligten Behörden über detaillierte Ausführungsregelungen immer häufiger Zielvorgaben/Zielvereinbarungen getroffen, die den Ausführenden Handlungsmöglichkeiten bieten, problemadäquatere Lösungen zu finden. Damit entsteht allerdings das Problem, dass nicht nur vertikal, sondern auch horizontal Inkompatibilitäten zwischen den Beteiligten erkennbar werden oder

1 Korte, K.-R.: Beschleunigte Demokratie: Entschleunigungsstress als Regelfall, in: Aus Politik und Zeitgeschichte 7/2012, $21 \mathrm{f}$..

2 Trankovits, L.: Verteidigung der Demokratie, in: Aus Politik und Zeitgeschichte 38-39(2012), 4.

3 Damit ist gemeint, dass sich Denk-Moden und Denk-Gemeinschaften herausbilden, die mehr oder weniger unkritisch gleiche Gedanken als wahr empfinden.

4 Nolte, P.: Von der repräsentativen zur multiplen Demokratie, in: Aus Politik und Zeitgeschichte 12/2011, 5-12. 
dass politisch-administrativ erarbeitete Vorstellungen anders umgesetzt werden als vom „Auftraggeber“ erwartet (principal-agent-Problem).

- Desgleichen wird Politik immer intensiver durch außerparlamentarische Kontrollen und Einflussmechanismen ,einem permanenten Durchleuchtungsprozess ausgesetzt, in dem auch die Grenzen zwischen Politischem und Privatem verschwimmen... ".5 Dabei kommt dem interaktiven Potential des Web 2.0 eine noch immer wachsende Bedeutung zu, bei gleichzeitig sinkender Bereitschaft der Bürgerschaft, sich intensiver mit politischen Prozessen zu befassen.

- Die Prognosesicherheit, mithin das Potential, die Auswirkungen politischer Entscheidungen zu erfassen, ist drastisch gesunken, nicht nur, weil es „,icheres Wissen" offenbar immer weniger gibt, sondern auch, weil die Entscheidungen sich auf Themen von großer Tragweite richten, die früher undenkbar waren (dies führte bekanntlich zu jener ubiquitär geführten Diskussion um „Risikogesellschaften“). Die Wirtschafts- und Finanzkrise im Rahmen der EU stellt hierfür ein gewisses Musterbeispiel dar, aber auch Entscheidungen über einen Ausstieg/Nichtausstieg aus der Kernenergie, Fragen zuzulassender oder nicht zuzulassender Modifikationen an Erbmaterial von Tieren und Pflanzen u.a.m. gehören dazu.

- Schließlich verkürzen sich die Fristen für Entscheidungsprozesse erheblich, da die schnellerlebige Welt auch schnellere Entscheidungen verlangt und langwierigen Beratungen in größeren Gremien Grenzen setzt. ${ }^{6}$

- Im Ergebnis spielen auch Veränderungen der politischen Kultur eine Rolle, wobei Medien, eine sich weiter pluralisierende Gesellschaftsstruktur und die Traditionen des Wohlfahrtsstaats Einstellungen befördern, den Staat (oder besser: die öffentliche Hand) für vieles verantwortlich zu machen und weniger auf Selbsthilfe zu setzen.

Was politische Prozesse besonders erschwert, sind die explodierenden Anspruchshaltungen, die seitens der handelnden Akteure immer weniger mit den verfügbaren Mitteln und Möglichkeiten befriedigt werden können. Diese Relation wäre für den politischen Prozess weniger gefährlich, wenn sich auch die gesellschaftlichen Einstellungen und Mentalitäten wandelten, etwa hin zu der Erkenntnis, dass der „Große Wurf“ im Rahmen politischer Prozesse nicht mehr oder nur selten möglich ist und dass es in den meisten Fällen eher um das ,[Backen] kleine[r] Brötchen“ oder eben um ein erweitertes , coping“ geht. $^{7}$

5 Kleinert, H.: Krise der repräsentativen Demokratie, in: Aus Politik und Zeitgeschichte 38-39(2012), 19 (mit Verweis auf John Keane).

6 Vgl. Rosa, H.: Beschleunigung. Die Veränderung der Zeitstrukturen in der Moderne, Frankfurt am Main, 2005.

7 Schimank, U.: Nur noch Coping: Eine Skizze postheroischer Politik, in: Zeitschrift für Politikwissen- 
Die schon frühzeitig diagnostizierte Überforderung der Politik/der ,,überforderte Staat ${ }^{\star 8}$ findet sich auf mindestens zwei Ebenen: auf der Ebene der Konsensfindung und jener der Risikobeherrschung. Überforderung ist aber ein eher subjektiver Begriff und insofern auch abhängig davon, ob und wie die Entscheidungsstrukturen institutionell und kognitiv befähigt sind, mit Überforderungen konstruktiv umzugehen. Unsicherheit belastet Politik umso mehr, je weiterreichend die Folgen von Entscheidungen und je weniger transparent diese sind bzw. je geringer das Wissen darüber ist. Gleichwohl gibt es subjektive Mechanismen der „Stress-Reduktion“. So fühlen sich Politiker offenbar weniger durch Unsicherheiten über das eigene Handeln unter Druck gesetzt, wenn die Öffentlichkeit anerkennt, dass Entscheidungen immer mit Unsicherheit behaftet sind, Fehlentscheidungen als Folge mangelnden Wissens vom Wähler und den Medien mithin als gleichsam ,höhere Gewalt“" verziehen werden. Zwar unterliegen die finanziellen Summen, die heute durch politische Entscheidungen bewegt werden, seit der ,Wende“ einem beunruhigend schnellen, fast inflationären Wachstum (waren früher Millionenbeträge die Referenzmarken, so begann sich die Öffentlichkeit ab 1990 an Milliardenbeträgen und seit dem Ausbruch der Finanzkrise sogar an Billionenbeträgen auszurichten), doch hat sich die Öffentlichkeit inzwischen daran gewöhnt, zumal die Zahlen eher abstrakt bleiben.

Schließlich bedrückt Politiker das „Entscheidbarmachen“ von Handlungsbedarfen angesichts der ausdifferenzierten Interessenlandschaft und der sich damit verbindenden Vielzahl von Akteuren, die bei wachsender vertikaler und horizontaler Verflechtung immer stärker auch Einfluss auf die Entscheidungsprozesse nehmen. ${ }^{9}$ Dabei sind Konsens- von Risikokosten zu trennen, obwohl die politische Praxis beides im Blick haben muss. Auf beide Aspekte reagieren die Akteure sehr sensibel; im Rahmen der Bewertung von Risikokosten tritt hinzu, dass Entscheider dazu tendieren, die Verlustrisiken weit höher einzuschätzen als die Gewinnchancen. ${ }^{10}$ Es ist zudem offen, ob Politiker bewusst zwischen Konsens-

schaft 21(2011), 455-463 (459 f.). Normativ gewendet schlägt Schimank sechs Strategien vor, die dem Regierungsstil Merkel ähneln: Keine Ziele setzen, an denen stur festgehalten wird; auf Gelegenheiten warten; Probleme aussitzen; irreversible Entscheidungen vermeiden; dem ausweichen, was unbedingt vermieden werden sollte; ,,abwarten und Tee trinken“.

8 Ellwein, Th./Hesse, J. J.: Der überforderte Staat, Baden-Baden, 1994/Frankfurt a.M., 1997.

9 Francis Fukuyama (Oh for a democratic dictatorship and not a vetocracy, in: Financial Times, 22.11.2011) hat in Reaktion auf die zunehmende ,vetocracy“ in den USA die Idee einer ,demokratischen Diktatur" - offenbar scherzhaft - in die Diskussion gebracht.

10 Bilgin, B.: Losses loom more likely than gains: Propensity to imagine losses increases their subjective probability, in: Organizational Behavior and Human Decision Processes, 118(2012), 203-15. 
und Risikokosten trennen, da höhere Risiken automatisch mit höheren Konsenskosten verbunden sind und diese wiederum gescheiterte oder verwässerte Entscheidungen begünstigen. Gleichwohl soll die Trennung hier beibehalten werden.

Nachfolgend wird erörtert, ob und in welcher Form sich die angesprochenen veränderten Reaktionsmuster der Politik tatsächlich herausgebildet haben. „Neu“ bedeutet dabei, dass sie sich von den bisher eingesetzten Verfahren und Politiken unterscheiden. Das heißt allerdings nicht, dass „das Bisherige verdrängt“, sondern ggf. eher ergänzend Berücksichtigung findet. Veränderungen sind in der Praxis häufig Überlagerungen von „Altem“ durch „Neues“, ohne dass das Alte sofort abstirbt. Allerdings wird es im Zeitablauf verdrängt, wenn sich veränderte Verhaltensmuster und Problemlösungen als effektiver, effizienter oder auch nur akzeptabler erweisen.

Methodisch richten sich die folgenden Aussagen auf deduktiv gewonnene Betrachtungen, nicht oder nur zum Teil an eigenen empirisch gehaltvollen Analysen aus. Die Informationsgrundlagen bilden sekundäranalytische Materialen: wissenschaftliche Veröffentlichungen, Medienberichte und Dokumente im Rahmen der Politikberatung.

\section{Erkennbare Reaktionen}

Die einfachsten Reaktionsmuster auf komplexe und schwierige Entscheidungssituationen bestehen darin, ggf. auf Nicht-Entscheiden auszuweichen oder der Entscheidung aus dem Weg zu gehen: durch symbolische Politik, durch Problemvertagung oder eine Problemverlagerung auf andere Entscheidungsträger. Probleme werden damit zwar angesprochen, ohne sie aber wirklich zu lösen, nicht selten mit dem Ziel, sie im Rahmen der öffentlichen Aufmerksamkeit ,herunterzuzonen".

Diese Reaktionsformen sind hier allerdings nicht gemeint. Es geht im Folgenden vielmehr um Entscheidungen, die tatsächlich getroffen werden müssen und mit erheblichen Kosten verbunden sind. Die Reaktionsmuster können dabei informatorischer und/oder materieller, formeller oder informeller sowie organisatorischer und/oder verfahrensbezogener Art sein. Da sie sich in der Regel auf institutionelle Kontexte richten oder sich mit ihnen zumindest auseinandersetzen müssen, werden vor allem informelle und verfahrensbezogene Reaktionen (,,institutionelle Reaktionen mit geringen politischen Kosten") behandelt. 


\section{Informelle Reaktionen}

Informelle Reaktionsformen sind eng mit informatorischen Verfahren verbunden: Man will Mehrheiten durch Überreden/Überzeugen gewinnen, dafür aber effektivere Modelle nutzen. Insofern sind informelle Reaktionen kaum von informatorischen Verfahren zu trennen.

\section{(a) Informelle Reaktionen auf Unsicherheit}

Bezogen auf das Problem wachsender Unsicherheit verfügt die Politik seit langem über Routinen, ihre Entscheidungssicherheit zu erhöhen. Damit sind Verfahrensweisen angesprochen, die das Risiko von Entscheidungen verringern sollen.

$\mathrm{Zu}$ den tradierten Verfahren gehört u.a., dass man Entscheidungen über den Einbezug von externem Sachverstand absichert, Expertengremien einsetzt (Enquête-Kommissionen, Sachverständigenräte, panels) oder Gutachten vergibt. Dazu zählt auch, über den Sachverstand von Fachorganisationen Vertrauen zu gewinnen, was meist noch immer im Rahmen unterschiedlicher Formen des lobbying bis hin zur Mitwirkung an der Erstellung von Gesetzes- oder Verordnungstexten reicht. Schließlich finden sich auch Formen, die Verantwortung zu diffundieren, etwa über eine erweiterte Beteiligung der Opposition. Allerdings ist der Zusammenhang zwischen Informationsverarbeitung und dem Einbezug machtvoller Entscheider sowie der Qualität von Entscheidungen nicht linear: Mehr Information bewirkt keineswegs automatisch bessere Entscheidungen, sondern zunächst nur höheres Selbstvertrauen im Entscheidungsverhalten. Zudem kann das erweiterte Machtbewusstsein die Entscheider dazu verführen, sich im Entscheidungsprozess zu selbstbewusst-optimistisch zu geben. ${ }^{11}$

Neuere Ansätze zur Risiko-Reduktion arbeiten vor allem mit Politiken der Risikostreuung, der Risikobegrenzung und der Risikoverlagerung. Risikostreuung dient der Diffundierung von Verantwortung, wofür sich Plebiszite, wo sie möglich sind, anbieten. Auch Planungsprozesse bieten hierfür viel Raum, zumal in diesem Kontext Öffentlichkeitsbeteiligungen aktiviert werden können. ${ }^{12}$ Eine andere Option bieten extensive Koordinationsbemühungen - durch den Einbezug

11 See, K. E./Morrison, E. W./Rothman, N. B./Soll, J. B.: The detrimental effects of power on confidence, advice taking, and accuracy, in: Organizational Behavior and Human Decision Processes, 116(2011), 272-85

12 Vgl. Forester, J.: The deliberative practitioner: Encouraging participatory planning processes, London, 1999 
zusätzlicher Akteure in die Verantwortung. ${ }^{13}$ Allerdings kann dies zu verzerrten Risikoeinschätzungen führen - sei es, dass Gruppen zur kollektiven Unverantwortlichkeit tendieren und eher riskantere Entscheidungen wagen (das gilt für den sog. risk shift der Gruppendynamik), sei es, dass die Entscheider, denen die Risiken schaden können, eher dazu neigen, negative Aspekte wesentlich höher als potentielle Gewinne einzuschätzen. ${ }^{14}$

Risikobegrenzung spielt in der Politik eine wichtiger werdende Rolle. Dazu gehören Experimentierklauseln und Befristungen von Gesetzen, jeweils verbunden mit der Auflage, die entsprechenden Regelungen zu evaluieren. Dazu gehören auch Modellvorhaben. Sie werden seit langem mit Erfolg initiiert, etwa über das Bundesamt für Bauwesen und Raumordnung (BBR). ${ }^{15}$ Gelungene Modellversuche reduzieren das Risiko und können Vetopositionen schwächen. Allerdings ist dieser Ansatz nur bei projektgebundenen Problemlösungen möglich. In besonders riskanten Situationen mit nicht gut zu prognostizierenden Handlungswirkungen wird eher mit der Strategie eines ,auf-Sicht-Fahrens“ entschieden, mit anderen Worten: mit einer Politik der kleinen Schritte. ${ }^{16}$ Auf-Sicht-Fahren sucht nach Lösungen in bestehenden Strukturen und verlängert damit häufig den Bestand von Fehl-Strukturen, mit nachfolgendem hohem Aufwand. ${ }^{17}$ Eine damit verwandte Strategie besteht darin, auf kleinteilige Lösungen zurückzugreifen: Statt „Reformen des großen Wurfs“ werden nur kleine, meist sektorale Änderungen angestrebt. Allerdings wird dabei die Risikobegrenzung nicht selten mit höheren Kosten erkauft, zudem wird den traditionellen Strukturen, dem status quo, sehr viel Bestand gelassen, sodass die Anpassungskosten den sich ändernden Rahmenbedingungen gegenüber in der Zukunft wachsen.

Risikoverlagerung schließlich bezeichnet eine früher sehr viel häufiger eingesetzte Strategie; so wurden Kernenergieanlagen bevorzugt an die Grenzen zum

13 Healey, P.: Planning through debate: The communicative turn in planning theory, in: Town Planning Review, 63(1992), 143-162; Healey, $P$ : Collaborative planning. Shaping places in fragmented societies, Basingstoke, 2. Aufl., 2006.

14 Bilgin, B., Losses, a.a.O., 203-15.

15 Vgl. Einig, K.: Funktionen und Folgen von Modellvorhaben für die Politikberatung, in: Informationen zur Raumentwicklung 7/8.2011, 435-52.

16 Dieses Verhalten ist selbst für Planer zu beobachten, die sich sonst gern als ,innovativ“ und ,progressiv“ darstellen: „Instead, they draw on established routines and practices to maintain order and predictability and to limit the potential for destabilising established processes and procedures" (Catney, $\mathrm{Ph} . /$ Hennebery, J.: (Not) exercising discretion: Environmental planning and the politics of blameavoidance, in: Planning Theory \& Practice, 13(2012), 551).

17 Eine der für Deutschland teuersten Strategien ist der Umgang mit der Euro-Krise. 
Nachbarstaat gelegt oder stationierten Gemeinden ihre Müllverbrennungsanlagen an den Grenzen zur Nachbargemeinde. In vereinfachter Form findet sich diese Vorgehensweise bereits in der politischen Figur des blame game beschrieben: Andere werden für Fehler oder Risiken verantwortlich gemacht. ${ }^{18}$ In gewisser Weise gehört dazu auch die Strategie des non-decision-making: Hier wird darauf verzichtet, Entscheidungen zu fällen, kommt es zu keinem aktiven Versuch, Risiken einzuschränken. Nicht zufällig ist non-decision-making häufig mit der Strategie der „Tadel-Vermeidung“ verbunden. ${ }^{19}$ Auch die bei Großprojekten zu beobachtende Neigung, die Kosten in der Entscheidungsvorlage zu niedrig anzusetzen und die Risiken zu verharmlosen, führt faktisch zu Risiko-Verlagerung auf die Zukunft und damit ggf. auf künftige Generationen.

Heute wird Risikoverlagerung sehr viel subtiler betrieben. ${ }^{20}$ Dies gilt etwa für die Verlagerung von Risiken auf ausgegliederte öffentliche Organisationen (etwa Gesundheitsbehörden) oder auch die Übertragung von Kreditausfallrisiken auf die EZB (statt sie nationalstaatlich auszuweisen). Generell ist zudem zu beobachten, dass versucht wird, Verantwortung vom Staat auf die Bürgerschaft zu übertragen, wozu auch die „Politik der Animation“21 zählt. Damit lassen sich Modelle anstoßen, wenn auch eher auf dezentraler Ebene, nicht zuletzt in Verfolgung von EU-Förderungen, die etwa im Rahmen der Strukturfonds einen solchen Ansatz nahelegen, zumal die EU-Kommission das „Partnerschaftsprinzip“ vorschreibt, um Risiken zu minimieren.

Mehr als früher ist man zudem bestrebt, Korrekturmöglichkeiten einzubauen. Diese sind vor allem dann relevant, wenn die Entscheidungen unter hoher Ungewissheit und Unsicherheit getroffen werden müssen. Gelegentlich bleibt den Politikern nur die Wahl unter Alternativen unterschiedlicher Fehlerintensität: „You are going to make mistakes, so you have to force yourself to decide which mistakes are easier zu correct".22

\section{(b) Informelle Reaktionen auf gewachsene politische Kosten}

Das Problem der Entscheidungsfähigkeit angesichts zahlreicher potentieller Veto-Positionen ist für Politiker allerdings wesentlich gravierender; sie werden

18 Hood, Chr: The risk game and the blame game, in: Government and Opposition, 37(2002), 15-37

19 Vgl. Weaver, R.K.: The politics of blame-avoidance, in: Journal of Public Policy, 6(1986), 371-398

$20 \mathrm{Vgl}$. Hood, C.: The risk game, a.a.O., $20 \mathrm{f}$.

21 Altrock, U.: Urban Governance in Zeiten der Schrumpfung, in: Heinelt, H./Vetter, A. (Hg.): Lokale Politikforschung heute, Wiesbaden, 2008, 301-26.

22 Timothy Geithner nach: Lessons learnt, in: The Economist, 19.01.2013, 48. 
daran gemessen, ob es ihnen gelingt, Mehrheiten für ihr Anliegen zu mobilisieren. Auch hier finden sich unterschiedliche Ansätze, dies zu betreiben.

Als tradierte Methoden gelten:

- Die Suche nach windows of opportunity, nach Gelegenheiten also, die vorhandenen Veto-Positionen zu schwächen - das ist primär eine Strategie für Reformen, die nicht an einen bestimmten Zeitpunkt gebunden sind.

- Die Nutzung eines erweiterten Ideen-Managements und der damit verbundenen Problemdefinition. Hier geht es darum, Probleme so zu definieren, dass sie im Rahmen des Machbaren bleiben und Politik als wirkungsvoll gilt, auch wenn wenig Inhaltliches damit verbunden ist. ${ }^{23}$ Diese Strategie ist weit verbreitet und kann über Varianten der Skandalisierung von Nicht-Reformen, einer Dramatisierung des Nicht-Entscheidens bis hin zu suggestiven Positiv-Formulierungen reichen. Agenda setting gilt ohnehin als wichtiges Instrument strategischer Politikgestaltung. ${ }^{24}$

- Der Ansatz, weitere Entscheidungen auf die Exekutive zu verlagern und das Parlament zu einem gleichsam nachträglichen Kontrollorgan zu degradieren. Dieser Prozess ist seit langem im Gang, wird in der Literatur immer wieder kritisiert und stellt auch grundsätzliche legitimatorische Fragen.

- Die Bildung von Vorentscheider-Strukturen (Informalisierung der Politik ${ }^{25}$ ), um sich aus komplexeren Entscheidungsstrukturen herauszuziehen bzw. diese nur noch formal in Anspruch zu nehmen.

- Bemühungen, sich quasi-plebiszitär zu verhalten und die Entscheidungsgrundlagen so zu ,frisieren“/zu dramatisieren, dass die Bevölkerung unmittelbar überzeugt wird, es gäbe nur diese und keine andere Entscheidungsmöglichkeit. Als klassisches Beispiel hierfür gilt die Emser Depesche Bismarcks. Neuzeitige „,plebiszitären Formen“ dieser Art werden verstärkt über „,Telepolitiken“ eingesetzt. ${ }^{26}$

- Die Aufbereitung von Entscheidungsgrundlagen über Experten, in der Hoffnung (zynisch: mit der Vorgabe), dass sich alle möglichen Alternativen als „nicht-gangbar“ (oder: ,alternativlos“) herausstellen; ihm korrespondieren Bemühungen, nach Möglichkeit ausländische Beispiele einzubeziehen und

23 Fry, T.: Politics as Design, Oxford u. New York, 2011, $187 \mathrm{ff} .$.

24 Vgl. Majone, G.: Agendy Setting, in: Moran, M/Rein, M./Goddin, R.E. (Hg.): The Oxford Handbook of Public Policy, Oxford, 2006, 228-50.

25 Florack, M./Grunden, T./Korte, K.-R.: Keine Governance ohne Government, in: Bröchler, S./v. Blumenthal, J. (Hg.): Regierungskanzleien im politischen Prozess, Wiesbaden, 2011, 181-202.

26 Vgl. Korte, K.-R.: Der Pragmatismus des Augenblicks. Das Politikmanagement von Bundeskanzler Gerhard Schröder 2002-2005, in: Egle, C./Zohlnhöfer, R. (Hg.): Endes des rot-grünen Projektes. Eine Bilanz der Regierung Schröder 2002-2005, Wiesbaden, 2007, 186 f. 
damit deutlich zu machen, dass man hinter dem Stand der Kunst zurückfallen würde, wenn man nicht in der gewünschten Weise entscheidet.

- Die Einholung hierarchischer Steuerung über die Schaffung von Entscheidungsgrundlagen durch höhere Ebenen; diese Funktion übernimmt im europäischen Kontext zunehmend die EU, während sie auf nationaler Ebene den Obersten Gerichten zugeschrieben wird,

- Die Entscheidung in Reaktion auf externe Risiken/externe Ereignisse, hier durchaus als äquivalent zur hierarchischen Steuerung. Damit erzeugt man Handlungsdruck (verbunden mit einer zeitlichen Begrenzung) und zwingt potentielle Veto-Spieler dazu, sich hinter die Entscheidung zu stellen, zumindest wenn erkennbar ist, dass es dazu keine machbare Alternative zu geben scheint (oder man diese nicht schnell genug mehrheitsfähig machen kann).

- Schließlich die Erhöhung des Entscheidungsdrucks und eine Begrenzung der Alternativensuche, indem man die Risiken des Nichtentscheidens dramatisiert und Terminzwänge setzt.

$\mathrm{Zu}$ den tradierten Routinen gehört auch, mögliche oppositionelle Einwände von vornherein auszuschalten. So kann man bei Großprojekten den Einwand zu hoher Kosten oder einer zu schlechten Kosten-Nutzen-Relation dadurch ausräumen, dass man Projektvorlagen üblicherweise mit zu niedrigen Kosten ansetzt resp. die Kostenschätzung auf einen optimalen Projektverlauf konzipiert, so dass die regelmäßigen Kostenüberschreitungen in der nachfolgenden Projektdurchführung zur Zeit der Projektentscheidung noch nicht sichtbar sind („Eisbergstrategie"). Oder die Nutzenseite wird über visionäre Szenarien so geschönt, dass Gegner es schwer haben, dagegen Argumente vorzutragen.

Neuere Ansätze im Umgang mit Problemen schwieriger werdender Konsensfindung bilden sich langsamer heraus, weil sie schnell auf institutionelle Hindernisse stoßen. Deshalb werden zunächst traditionelle strategische Elemente weiterentwickelt, insbesondere das Management von Ideen und das angesprochene agenda setting. Hier kommt den Regierungen zugute, dass die Komplexität der Entscheidungsfelder Möglichkeiten für symbolische Politik eröffnet, auch für politische story lines (oder auch Narrative) sowie generell für paradigmatische Steuerung, weil sich immer weniger Akteure zutrauen, die Dinge noch durchschauen zu können. ${ }^{27}$ Vielfach genügen politische Signale, um in der Wirtschaft, vor allem den immer bestimmenderen Finanzmärkten, Entscheidungen zu begünstigen. 
Um größere institutionelle Änderungen zu vermeiden, werden informelle institutionelle Modifikationen bevorzugt. ${ }^{28}$ Diese Art der Informalität zeigt sich bereits bei der Entscheidungsvorbereitung über die vorab-Einbindung wichtiger Entscheidungsträger, einschließlich wichtiger Vetogruppen. Das wird von den Politik- und Verwaltungswissenschaften allerdings mit gemischten Gefühlen verfolgt, da solche außerparlamentarischen Diskussionsforen faktisch politische Entscheidungen vorprägen, ohne dafür ausreichend politisch legitimiert zu sein. Zudem verändern sie Machtstrukturen, weil Akteure einflussreich werden, die die formalen Strukturen aus guten Gründen eher zurückdrängen.

Ebenfalls noch innerhalb bestehender institutioneller Kontexte findet sich die Bedeutung gewinnende Strategie, einige Parameter für künftiges Handeln festzuschreiben und dann nach Lösungen zu suchen, wie innerhalb dieser veränderten Ausgangssituation neue Entwicklungen gestaltet werden können. Das ist allerdings ein „kühnes“ Vorgehen, vor allem dann, wenn unklar ist, wie die Lösungen konkret aussehen können und ob sich überhaupt Lösungen finden, die den alten Zustand sinnvollerweise ablösen können. Beispiele dafür sind die in Niedersachsen (allerdings geglückte) Entscheidung, die Bezirksregierungen abzuschaffen (2005 unter Christian Wulff ${ }^{29}$ ) sowie, aus neuerer Zeit, die Entscheidung der Bundesregierung, nach der Katastrophe von Fukushima den Ausstieg aus der Kernenergie einzuleiten, zu beschleunigen und in großem Ausmaß auf erneuerbare Energie zu setzen. Auch die Entscheidung, die allgemeine Wehrpflicht abzuschaffen, ist in diese Richtung zu interpretieren.

Als „kraftvoller“ (und weniger problembesetzt) gilt das Verfahren, mit den zu beteiligenden Akteuren Zielvereinbarungen zu treffen, die möglichst mit einer Vereinbarung über die nachfolgenden Umsetzungsschritte untermauert werden (road mapping). Die Wirksamkeit dieses Vorgehens setzt allerding einen kollektiven Lernprozess voraus, das Problem als gemeinsames anzuerkennen und zu einem Beitrag bereit zu sein.

Mitunter werden handlungsleitende Rahmenbedingungen extern vorgegeben, durchaus unter Einfluss dessen, der „steuern“ will. Solche Formen der „hierarchischen Steuerung" werden seit Gründung der EU vermehrt über die Europäi-

28 Manow, Ph.: Informalisierung und Parteipolitisierung. Zum Wandel exekutiver Entscheidungsprozesse in der Bundesrepublik, in: Zeitschrift für Parlamentsfragen 27(1996), 96-107.

29 Grabowski, P.: Das neue Kleid der Landesverwaltung: die Zweistufigkeit, in: Niedersächsische Verwaltungsblätter 12/2006, 328-30; ausführlich bei Hesse, J.J.: Der Staat in der Fläche: Landesverwaltung ohne Mittelinstanz?, Baden-Baden, 2011, sowie ders.: Handlungs- und zukunftsfähige Kommunalstrukturen. Der Fall Niedersachsen, Baden-Baden, 2011. 
sche Kommission eingeleitet, die solche Regeln zwar zu setzen vermag, hier aber zunehmend auf den Widerstand nicht nur der Mitgliedstaaten, sondern auch der „Völker Europas“" trifft.

Da die Setzung von Rahmenbedingungen mit weiter reichenden Konsequenzen für zukünftiges Handeln riskant ist, neigen Regierungen dazu, stattdessen lieber (unverbindliche) langfristige Zielparameter vorzugeben. Werden sie nicht erreicht, kann das Versagen auf eine Reihe nicht-kontrollierbarer Einflussfaktoren zurückgeführt werden oder sucht man andere Regierungen dafür verantwortlich zu machen. Ein gutes Beispiel bildet die Erklärung der Regierung Schröder, bis 2020 einen Flächenverbrauch von täglich durchschnittlich 30 ha zu erreichen (2012 noch über 70 ha). Solche längerfristigen Zielkorridore, die eher Handlungsrichtungen vorzeichnen als klare Zielmarken zu definieren, lassen sich mit einer „Politik der kleinen Schritte“ verbinden: Die Auswahl und Abfolge der Schritte folgt einer Logik, die für Außenstehende jedoch nicht erkennbar sein muss. Kleine Schritte haben den Vorteil, die Konsensfähigkeit zu erhöhen und gleichzeitig das Risiko der Entscheidung zu mindern. Faktisch ist der Ansatz demjenigen ähnlich, vollendete Tatsachen zu schaffen, die weiteres Handeln immer stärker in Richtung eines brinkmanship ${ }^{30}$ drängen; im Ergebnis gibt es „,kein Zurück mehr“.

Es ist nicht verwunderlich, dass diese letztbenannte Strategie vor allem im Bereich der Umweltpolitik eingesetzt wird. Denn hier kann man von hierarchischer Steuerung durch die EU profitieren, die von allen Mitgliedstaaten als „Gemeinschaftsaufgabe“ anerkannt wird. Entsprechende Zielansagen sind zudem verbindlich, wenn sie über EU-Richtlinien verabschiedet werden. Im Gegensatz dazu haben Ziele, die von Bundes- und Landesregierungen festgelegt werden, häufig nur orientierende Funktion und bleiben im Fall der Nicht-Erreichung sanktionsfrei.

Besonderen Konsensbedarf verlangen Themen mit hohen Verteilungswirkungen. Sie verhindern Strukturreformen und weiterreichende Innovationen. Traditionell werden verteilungsorientierte Entscheidungen aufgeschoben, dann längerfristig aufgebaut und mit Handlungszwängen verbunden, denen auch politische Gegner nicht ausweichen können (erneut: ,alternativlos“). Ein neuerer strategischer Ansatz zur Lösung von Verteilungsfragen geht dahin, Verteilungsfragen mit

30 Unter brinkmanship versteht man eine (Verhandlungs-)Strategie, bei der man eine Situation für den Gegner immer bedrohlicher macht, gleichzeitig aber auch erkennen lässt, dass es kein Zurück mehr gibt. Ziel ist es, den Gegner dadurch zu Zugeständnissen zu veranlassen. 
Entwicklungsfragen zu verknüpfen. Die Grundidee ist die Stärkung des Subsidiaritätsprinzips und der Grundsatz des „Forderns statt Förderns“. Auf dieser Grundlage werden immer häufiger Wettbewerbsverfahren bei der Mittelvergabe eingesetzt, ${ }^{31}$ zumindest dann, wenn diese möglich und begründbar sind (etwa bei Subventionen für die Wirtschaft), nicht hingegen dort, wo Transfers eine reine Ausgleichsfunktion wahrnehmen. Damit verbindet sich auch die Hoffnung, Strukturreformen leichter durchsetzen zu können, weil die Zahl der status-quoVerteidiger reduziert wird.

„Harte“ Konflikte werden zudem immer häufiger über Mediationsverfahren zum Ausgleich gebracht. ${ }^{32}$ Sie sind zwar nicht grundsätzlich neu (Streitschlichtungsverfahren im Privatrecht, Schlichtungsverfahren im Tarifrecht), doch wurden sie bei Auseinandersetzungen im Rahmen politischer Konflikte bislang kaum genutzt. Hier setzt man eher auf die angesprochenen Partizipationsverfahren, deren Wirksamkeit sich jedoch im Konfliktfall als sehr begrenzt erwiesen hat.

\section{Institutionelle Reaktionen mit geringen politischen Kosten (Verfah- rensänderungen)}

Da die bislang angesprochenen Reaktionen immer weniger ausreichen, den veränderten Rahmenbedingungen gerecht zu werden, wächst der Druck auf Politik und Gesellschaft, sich den neuen Entscheidungskontexten auch institutionell anzupassen. Institutionelle Änderungen beziehen sich dabei auf Organisationsund Verfahrensanpassungen, wobei Änderungen mit geringeren politischen Kosten (da sie die Machtstrukturen nicht ostentativ verändern) bevorzugt werden. Es handelt sich im Wesentlichen um verfahrensbezogene Änderungen (formeller und informeller Art), auf die im Folgenden verstärkt eingegangen wird. Erneut kann hier nach Konsens- und Risikokosten unterschieden werden.

\section{(a) Entscheidungsentlastung}

Zur Entscheidungsentlastung gehören die Konzentration von Entscheidungen auf das Kabinett ${ }^{33}$ oder das „Stille Regieren“ über Machtzirkel, ${ }^{34}$ aber auch die um-

31 Benz, A.: Leistungswettbewerbe in der regionalen Raumentwicklungspolitik, in: DISP 157(2004), 4-10.

32 Ury, W.: Getting past no. Negotiating with difficult people, London 1991. In Deutschland wurde dazu 1992 der Bundesverband Mediation e.V. gegründet.

33 Falter, W./Müller, K.: Graue Eminenzen der Macht. Küchenkabinette in der deutschen Kanzlerdemokratie. Von Adenauer bis Schröder, Wiesbaden 2004

34 Korte, K.-R.: Der Pragmatismus des Augenblicks, 2007, a.a.O., $181 \mathrm{f}$. 
fangreiche „Netzwerkpflege“ und die Auslagerung von Vorentscheidungen auf Gremien. Selbst die Externalisierung der Politikformulierung ist dem deutschen politisch-administrativen System nicht mehr fremd (Gesetzgebung auf Honorarbasis $^{35}$ ). Für diese Form der Politikvorbereitung und -formulierung wurde vor allem die Regierung Schröder bekannt. Im Ergebnis kam es durchaus zu Veränderungen von Macht- und Einflussstrukturen, deren Ausmaß und Wirkung allerdings wenig transparent blieben.

Formelle Verfahren der Reduktion der Entscheiderzahl sind seltener. Zwar findet sich seit langem die Tendenz, immer mehr Entscheidungen mit Argumenten der kurzen Entscheidungsfristen und der Geheimhaltungsbedarfe auf die Exekutive zu übertragen, eine Argumentation, die allerdings verfassungsrechtlich zunehmend als bedenklich eingestuft wird. ${ }^{36}$ Neu ist es zudem, auch innerhalb des Parlaments die Zahl der Entscheider mit Verweis auf Eilbedürftigkeit und Vertraulichkeit zu begrenzen - wie der gescheiterte Versuch des Bundestags und der Bundesregierung dokumentierte, Entscheidungen zum Europäischen Stabilisierungsmechanismus auf ein aus Mitgliedern des Haushaltsausschuss gewähltes Gremium (neun Abgeordnete) zu übertragen (BVerfG, Urteil v. 28.02.2012, 2 BvE 8/11).

Eine weichere, eher auf Verfahren bezogene Methode wird seit längerem seitens der EU genutzt - die sog. Offene Methode der Koordinierung, ${ }^{37}$ die über ein Kaskadensystem immer konkreter werdender Entscheidungen bei immer kleinerer Entscheiderzahl und geringerem Entscheidungsspielraum den Entscheidungsprozess positiv zu gestalten sucht. Diese Methode hat keine verbindliche Wirkung, soll aber Lernprozesse auslösen und konsens- wie effizienzorientiertes Handeln unterstützen. ${ }^{38}$

35 Döhler, M.: Gesetzgebung auf Honorarbasis, in: Politische Vierteljahresschrift 53(2012), 181-210.

36 Vgl. Urteil des Bundesverfassungsgerichts vom 09.06.2012 (BVerfG, 2 BvE 4/11).

37 Bernhard, St.: Die offene Methode der Koordinierung als Prozess von Lernanreizen - zu Theorie und Praxis der europäischen Inklusionsstrategie, Bamberg 2005 (Bamberger Centrum für Europäische Studien, Discussion Paper No. 8/2005).

38 Auf EU-Ebene werden gemeinsame Ziele resp. guidelines und Verfahren vereinbart, die jedes Mitgliedsland nach eigenen Bedingungen umsetzen muss, wobei der Umsetzungsprozess und sein Ergebnis anhand von Indikatoren gemessen und beurteilt werden kann. Die Mitglieder unterliegen Berichtspflichten, auch soll durch Veröffentlichung der regelmäßigen Evaluierungen ein transparenter Prozess organisiert werden, der durch sozialen Druck auf der nationalstaatlichen Ebene verstärkt werden könnte. Kritik an dem Verfahren richtet sich auf drei Punkte: die (nicht-demokratische) Zielfestlegung, die (technokratische) Festlegung der Indikatoren und die schwache Wirkung gegenüber starken Interessen (nicht rechtsverbindliches Verfahren). 


\section{(b) Risikoentlastungen}

Am weitesten verbreitet sind dagegen Verfahren zur Verantwortungsdiffusion, die es zwar schon immer gab, heute aber differenzierter eingesetzt werden. Dem dienen u.a. Beteiligungsformen, über die man Entscheidungsprozesse stärker zu dezentralisieren sucht. ${ }^{39}$ Damit wird angestrebt, die Verantwortung auf weitere gesellschaftliche Gruppen zu verteilen. Freilich verbindet sich mit solchen Ansätzen häufig auch eine Entscheidungsüberlastung, vor allem dann, wenn man die zu Beteiligenden lediglich in beratender Funktion einbindet. Auf diesem Weg werden Quasi-Mitentscheider geboren, die zwar Machtstrukturen verändern können, aber keine politische Verantwortung tragen. Auch Bemühungen, der Bevölkerung zusätzliche Mitsprachemöglichkeiten zuzugestehen, sind unter dieser Rubrik zu verbuchen. Wenn es aber gelingt, Aufgaben komplett zu delegieren und damit u.U. die Politikverflechtung zwischen den Ebenen zu reduzieren, könnte dies vorteilhaft sein - doch zeigt die Praxis, dass faktisch der umgekehrte Weg beschritten wird: Die Politikverflechtung nimmt überall zu, multilevel-Prozesse werden zum Standard. Dahinter verbirgt sich wohl auch das Bemühen von Politikern, die Kontrolle über Entscheidungsprozesse nicht gänzlich aus der Hand zu geben, weshalb auch die Erfordernisse für direktdemokratische Entscheidungen extensiv definiert werden (Quoren etc.), sodass bei den bereits gegebenen Möglichkeiten ,,viele Verfahren ....an hoch angesetzten Erfolgshürden" scheitern und deshalb einer Herrschaft durch Ämterhäufung und Verweildauer in politischen Funktionen nicht wirklich entgegenwirken können. ${ }^{40}$

Im Übrigen werden Bürgerentscheide (Plebiszite) immer wieder als ein „Heilmittel“ diskutiert, ${ }^{41}$ weil sie zwei Vorteile versprechen: den Einbezug der Bevölkerung in die Mitverantwortung für Entscheidungen und die Riskoentlastung durch Verantwortungs-Delegation. Aber sie lassen sich nur für ganz bestimmte, binär entscheidbare Themen (ja-nein-Entscheidungen) nutzen und sind für komplexe Probleme schlecht geeignet (haben die zur Entscheidung Aufgerufenen das notwendige Wissen und die erforderliche Ernsthaftigkeit für ihre Entscheidung, wenn sie faktisch dafür nicht haftbar gemacht werden können? ${ }^{42}$ ). Zudem sind

39 Vgl. Collet, St./Tillmann, Ch./Schwickert, D.: Der Bürger als neuer Akteur der Politikberatung Schlussfolgerungen und Erfolgsfaktoren, in: Bertelsmann-Stiftung, Hg.: Wie Politik von Bürgern lernen kann. Potenziale politikbezogener Gesellschaftsberatung, Gütersloh 2011, 200-214.

40 Bender, Ch./Wiesendahl, E.: „Ehernes Gesetz der Oligarchie“: Ist Demokratie möglich? in: Aus Politik und Zeitgeschichte 44-45/2011, 24.

41 Vgl. Jung, S.: Die Logik direkter Demokratie, Wiesbaden 2001.

42 Vgl. Trankovits, L.: Verteidigung der Demokratie, in: Aus Politik und Zeitgeschichte 38-39/2012, 3-6; 
sie bei raschem Entscheidungsbedarf wenig hilfreich. Schließlich führen sie nicht selten zu strukturell bedingten Verengungen der Problemdefinition und erweisen sich für gut organisierte Sonder-Interessen offen, die sich ihre Mehrheiten besorgen. Auch ist zu bedenken, dass Bürgerentscheide häufig nur vorläufige Entscheidungen sind, weil das Thema bei ablehnendem Ergebnis nach einer Zeit wieder zur Entscheidung vorgelegt werden kann und dem Gesetzgeber bei Zustimmung ggf. die Möglichkeit der Revision offen steht. Schließlich sind direktdemokratische Verfahren nicht unbedingt demokratischer, sondern eher sozialselektiv, werden von einer relativ kleinen Gruppe bestimmt (Wahlbeteiligung unter $40 \%$ ) und sind insofern nicht frei von verzerrten Interesseneinflüssen. ${ }^{43}$ Der faktische Vorteil der Bürgerentscheide ist der damit verbundene breite Diskurs über ein politisches Thema - auch damit kann Politik entlastet werden.

Beliebt sind schließlich institutionelle Änderungen gleitender Art, die mangels dezidierter politischer Entscheidungen zur Entscheidungsverlagerung auf (vermeintlich) entpolitisierte Gremien (Verfassungsgericht, Zentralbank) oder auf Organisationen außerhalb des Staates führen - wie die Verlagerung der Lösung der Euro-Krise auf die Europäische Zentralbank unter Präsident Draghi. Hier wurde durch die Öffnung der Zentralbank für die Schuldenfinanzierung faktisch ein Politikwechsel zur sog. Transferunion eingeleitet, den deutsche Politiker vermeiden wollten, weil er - zumindest in Deutschland - nicht durchsetzungsfähig gewesen wäre. ${ }^{44}$ Eine Tendenz zur „Expertokratie“ ist darin unverkennbar. Heute formieren sich in zahlreichen Ländern Parteien, die sich in Reaktion hierauf für eine verstärkte Mitsprache der Bevölkerung einsetzen und sich gegen „Eliten-Herrschaft“ und die politische Suggestion von „Alternativlosigkeit“ in Entscheidungssituationen wenden.

Darüber hinaus wird die politische Entscheidung von den Trägern nicht selten auf ad-hoc-Gremien verlagert, die weniger politisiert und stärker sachorientiert arbeiten sollen. $\mathrm{Zu}$ unterscheiden sind solche Strategien allerdings von der angesprochenen symbolischen Politik - die auch über entsprechende Gremien laufen kann, wobei allerdings von vornherein deutlich ist, dass keine entscheidungsreifen Lösungen produziert werden; im Ergebnis wird damit Zeitgewinn und Problemvertagung erkauft. Im Übrigen wird über die Bildung von Arbeitskreisen

Kleinert, H.: Krise der repräsentativen Demokratie, in: Aus Politik und Zeitgeschichte 38-39/2012, 1824.

43 Merkel, W.: Volksabstimmung: Illusion und Realität, in: Aus Politik und Zeitgeschichte 44-45/2011, 55. 44 Vgl. hierzu die einschlägigen Beiträge in den Jahrgängen 2011 bis 2013 der ZSE. 
heute Politik gern vorgetäuscht: „,Task-Force‘ ist in Washington ein anderer Ausdruck dafür, dass die Regierung vorgibt, etwas zu unternehmen, während sie das Thema tatsächlich in die runde Ablage befördert ${ }^{\star 45}$ Es verstärken sich allerdings die Bemühungen, solche Arbeitskreise und focus groups dafür einzusetzen, Entscheidungen tatsächlich vorzubereiten - und zwar auf hohem Wissensniveau, weniger ideologisch besetzt und intersektoral koordiniert. Die amerikanische Politik nutzt im Rahmen ihres Zwei-Parteiensystem gelegentlich bipartisan commissions, um Entscheidungen, die auf regulärem Wege nicht zu erreichen wären, über diese institutionalisierte Form der Vorentscheidung zu ermöglichen oder doch zumindest zu skizzieren.

\section{Wachsende Lernfähigkeit der Politik}

Politische Strategien werden meist nicht unkontrolliert und zufällig gewählt, sondern von Politikern bewusst genutzt. Dabei geht es allerdings nicht nur darum, politische Kosten zu vermeiden oder zu verringern, es kommt vielmehr auch darauf an, die politische Entscheidungsfindung effektiver zu machen. Politik ist heute immer stärker gefordert, sich nicht nur um effektives Politikmanagement zu kümmern, sondern für neue Herausforderungen oder neue Rahmenbedingungen auch neue Lösungen zu finden. Ein wesentlich größeres Gewicht als früher wird deshalb auf individuelles wie kollektives Lernen gelegt. Lernen bezieht sich nicht nur auf Veränderungen von Denkmustern und Einstellungen, sondern auch auf die Übernahme von bewährten Problemlösungen aus anderen Staaten/anderen Handlungssystemen. Denn auch darüber lassen sich kollektive Lernprozesse organisieren, weil die guten Beispiele das Imitationslernen fördern, gleichzeitig aber auch Vetopositionen schwächen. Allerdings ist diese Form des Lernens aufwändig, selten finden sich Vorbilder, die eins-zu-eins übernommen werden können. So wird Überzeugungsarbeit notwendig, um etwaige Lösungen der Problemsituation angemessen zu gestalten und es den eigenen Institutionen zu erlauben, sie zu übernehmen, falls sich geeignetere Alternativen finden. ${ }^{46}$

Inzwischen sind Lernprozesse aus der politischen Entscheidungsfindung nicht mehr wegzudenken, zumal über den hohen Anteil akademisch ausgebildeter

45 Reich, R.: Superkapitalismus. Wie die Wirtschaft unsere Demokratie untergräbt, Frankfurt/New York 2008, 263.

46 Policy-Learning erfolgt in der Praxis allerdings sehr viel differenzierter, weil es immer auch auf die Transaktionskosten, die Gelegenheiten zum Lernen, die Netzwerke für Informations- und Know-HowAustausch etc. ankommt (vgl. Shipan, Ch. R./Volden, C.: Policy diffusion: Seven lessons for scholars and practitioners, in: Public Administration Review 72(2012), 788-96). 
Politiker der Wissenstransfer in die Praxis beschleunigt wurde. Lernprozesse erfolgen jedoch bei unterschiedlichen Themen und unterschiedlichen Akteuren unterschiedlich schnell, was die Konsenskosten erhöht. Im technischen Bereich ist das Lernen (bereits institutionell begründet) relativ ausgeprägt - hier wirken der Wettbewerb von Forschungseinrichtungen, der globale Wettbewerb im Unternehmensbereich und die Öffnung von Forschungsministerien für Neuerungen mit. ${ }^{47}$ Veränderungen mit erheblichen sozialen Folgen vollziehen sich demgegenüber langsamer - am langsamsten bei den (Negativ-)Betroffenen selbst. Um hier eine bessere zeitliche wie materielle Abstimmung zu erreichen, werden politische Entscheidungen gern mit framing-Prozessen verbunden. Darunter versteht man die Beeinflussung von Einstellungen und Denkmustern über Paradigmata, die mehrheitsfähig gemacht werden. ${ }^{48}$ So war die Agenda 2010 wesentlich mitbeeinflusst durch die vorausgehende, von Wirtschaftskreisen angestoßene Diskussion um die Gefährdung des Standortes Deutschland im internationalen Wettbewerb. Im Gefolge dieser kampagnenartigen Debatte, die von den Medien unterstützt wurde, konnten Vetogruppen sich immer weniger durchsetzen und musste die Regierung schließlich eine neoliberal inspirierte Politik der Arbeitsmarktreform initiieren.

Lernprozesse lassen sich durch geeignete Verfahren unterstützen. Auf nationaler Ebene werden Konsensbildungsprozesse immer häufiger über Modellvorhaben, öffentliche Diskurse, Vergleiche mit anderen Ländern (z.B. Vergleichsstudien wie PISA oder Ranking der nationalen Standortqualitäten ${ }^{49}$ ) u.ä. vorbereitet. Ob es sich dabei immer um Lernprozesse handelt, ist strittig. Teilweise sind es Prozesse, welche die Vetopositionen durch überzeugendere Argumente schwächen: Wenn Modellvorhaben, Vergleiche mit dem Ausland, Aufforderungen der OECD und internationale Rankings von der Politik aufgegriffen werden, schwächen sie die Argumentationskraft der Vetostimmen.

Lernprozesse werden schließlich auch über „Szenario-Analysen“ unterstützt. Immer häufiger sucht man die Zukunft über Szenarien ,entscheidungsfähig“ zu machen - auch wenn damit in manchen Fällen schlicht die Bandbreite möglicher

47 Vgl. Marz, L.: Innovation als Valorisierung. Die Karriere der Wasserstoff- und BrennstoffzellenTechnologie in Deutschland von 1970-2010. Eine Fallstudie, Berlin 2010 (WZB-Discussion Papers, SP III 2010-402).

48 Vgl. Surel, Y: The role of cognitive and normative frames in policy-making, in: Journal of European Public Policy 7(2000), 495-512.

49 Wie vom Institut der deutschen Wirtschaft (Köln), der OECD oder dem World Economic Forum (Davos) regelmäßig durchgeführt. 
Entwicklungen eingegrenzt wird. Szenarien haben aber den Vorteil, relativ plastisch vor Augen zu führen, welche Auswirkungen sich mit einer Entscheidung verbinden. Dies kann Fluch und Segen zugleich sein - damit können Befürworter gewonnen werden, aber umgekehrt mehren sich auch diejenigen, die sich von den Negativwirkungen betroffen fühlen. Das ist vor allem dann problematisch, wenn sich Medien einmischen und relativ früh für eine der Seiten plädieren, weil sie glauben, ihre Klientel teile mehrheitlich diese Meinung.

Zum policy-Lernen gehören zunehmend auch Wirkungsanalysen. Gemeint sind damit nicht nur Analysen bezogen auf wiederkehrende Entscheidungen, sondern auch solche, die sich auf noch nicht erprobte Neuerungen beziehen: Werden die Wirkungsanalysen zum ständigen Begleitprozess des Entscheidungshandelns, gleichsam als Maßnahmen-controlling ausgestaltet, so erhöhen sich die Chancen, das „Auf-Sicht-Fahren“ mancher Entscheidungen rationaler zu gestalten, aber auch, politische Entscheidungen stärker reversibel zu halten. Man erkennt Fehlentwicklungen früher und kann die Maßnahme abbrechen, wenn es notwendig wird. Den dienen auch jene Vergleichs-Untersuchungen, die spätestens seit Einsetzen der Föderalismusreform-Kommission II und der nachfolgenden Erweiterung des Grundgesetzes (Art. 91d) in die deutsche Politik einzuziehen beginnen. Dies wiederum wird ergänzt durch Handreichungen praxisorientierter Wissenschaft, wie sie etwa in der inzwischen vorgestellten und durchgeführten „Strukturberichterstattung für die Gebietskörperschaften“ ihren Ausdruck findet. ${ }^{50}$

Auch der sog. wachsende Pragmatismus in der Politik, der dazu führt, dass sich die Parteien in ihrer praktischen Politik immer mehr annähern, lässt sich als „Lernen“ interpretieren: nicht zuletzt durch die Anerkennung, dass der nationale Gestaltungsspielraum für ideologisch geprägte Lösungen kleiner geworden ist und es sich empfiehlt, Mehrheiten über Parteigrenzen hinweg zu organisieren.

\section{Zum Zusammenhang zwischen politischen Kosten und Gestal- tungsmacht}

Der Wandel der Politikmodi lässt sich zwar so interpretieren, dass Politiker sich primär an politischen Kosten, also Konsenskosten und Risikokosten, orientieren, es in der praktischen Politik aber vor allem auch darum geht, die Gestaltungsmacht zu erhöhen und den politischen Gestaltungsspielraum zu erweitern. Denn

50 Programmatisch hierzu Hesse, J.J.: Der Bund in der Verantwortung: Plädoyer für eine nachhaltige Modernisierung von Regierung und Verwaltung, in: ZSE 5/1 (2007), 99-111. 
Reduktion politischer Kosten ist häufig gleichbedeutend mit Mehrung der Gestaltungsmacht. Das lässt sich aufzeigen, wenn man genauer betrachtet, wie politische Kosten reduziert werden.

\section{(a) Konsenskosten}

Konsenskosten lassen sich über zwei unterschiedliche Ansätze einschränken: über die Zahl der Entscheider und über die Modifikation des Entscheidungsbedarfs. Ob der eine oder andere Ansatz im Vordergrund steht, hängt zunächst von der Art der Themen und den institutionellen Rahmenbedingungen ab. Sehr einflussreich sind zudem die Politikstile, die stärker subjekt- oder stärker objektbezogen sein können. Subjektbezogene Politikstile nutzen vor allem Vorentscheiderstrukturen und Netzwerke der wichtigen Entscheider, während objektbezogene Politikstile sich mehr auf die Themen- und Alternativenmanipulation richten (Ideenmanagement, Problemdefinition, Optionenausschluss etc.).

Die Zahl der Entscheider lässt sich primär institutionell reduzieren, die Zahl der Alternativen und Themen dagegen informatorisch und normativ. Informatorische Methoden können allerdings auch den Einfluss von Entscheidern modifzieren (Entwertung von Vetopositionen) oder Meinungskonformismus herausbilden (groupthink ${ }^{51}$ ).

Die institutionelle Verringerung der Zahl der Entscheider erfolgt primär dadurch, dass Entscheidungsprozesse hierarchisiert oder dezentralisiert, Vorentscheiderstrukturen geschaffen oder Entscheidungen in Gremien mit geringeren politischen Kosten verschoben werden. Es gelingt aber auch, opponierende Entscheider in ihrem Einfluss zu reduzieren, indem sie marginalisiert werden - bis hin zu einem Punkt, an dem sie von der Öffentlichkeit unter Druck gesetzt werden können. Besonders das Internet gewinnt über seine sozialen Netzwerke wachsende Bedeutung, vor allem über Formen der sog. Schwarm-Intelligenz: Die inzwischen sattsam bekannten sog. shitstorms lassen sich leicht und schnell organisieren, samt des sich damit verbindenden moralischen und politischen Drucks auf Entscheider. Ob sie letztlich wirksam sind, ist angesichts des Mangels belastbarer wissenschaftlicher Erkenntnisse noch nicht abschließend $\mathrm{zu}$ beurteilen. ${ }^{52}$ Zudem sind „Schwärme“ in der benannten Form nicht ungefährlich, weil sie nur schwer von dem einmal eingeschlagenen Weg abzulenken sind.

51 Janis, I. L.: Victims of groupthink. Psychological studies of policy decisions and fiascoes, 2. erw. Aufl., Boston 1982.

52 Untersuchungen zu outcomes of social movements and protest wurden am WZB (Berlin) durchgeführt (WZB-Mitteilungen 134/2011, 53 f.). 
Gestaltungsmacht lässt sich schließlich auch über informatorische Instrumente mehren. Sie lassen Deutungshoheit über Problemfelder gewinnen, häufig auch mit dem Ziel, die Zahl der in die Entscheidung einbezogenen Alternativen zu vermindern und die Zahl der zu entscheidenden Themen zu verringern. Letzteres gelingt etwa dadurch, dass man Themen herunterspielt oder als nicht politisch, sondern marktlich zu lösen ansieht, sie ggf. auch in die Privatsphäre abzuschieben sucht.

\section{(b) Risikokosten}

Risikokosten stellen für Politiker ein besonderes Problem dar, weil sie schlecht beherrschbar sind. Deshalb sind politische Funktionsträger primär daran interessiert, Risiken zu vermeiden oder zumindest zu minimieren. In welchem Maß das erforderlich ist, wird allerdings auch von der politischen Kultur eines Landes mitbestimmt - je risikoempfindlicher eine Gesellschaft ist, um so mehr versuchen Politiker, Risiken zu vermeiden. ${ }^{53}$

Zur Minderung von Risikokosten gibt es prinzipiell vier Möglichkeiten:

- Verbesserung der Informationsgrundlage. Hier geht es darum, die Wirkungen von Entscheidungen transparenter zu machen. Vermehrt wird dabei auch versucht, die angesprochene Schwarm-Intelligenz konstruktiv zu nutzen. ${ }^{54}$ Dass es auch dabei nicht nur um die Minderung der politischen Kosten, sondern auch um die Gestaltungsmacht geht, zeigt im Übrigen die zunehmende Bedeutung von Diskursen in Experten-Gruppen, gelegentlich auch in der Version der angesprochenen focus groups. ${ }^{55}$

- Breitere Risikostreuung und Verlagerung auf zusätzliche Akteure (RisikoDiffundierung). Dazu gehört auch, die Zahl der Risikoträger über Wettbewerbsverfahren zu erhöhen. Durch Vorgaben von Rahmenbedingungen, aber durch kreativitätssteigernde Wettbewerbsverfahren wird versucht, die Risiken konstruktiv zu streuen, d.h. gleichzeitig neue Lösungen zu finden, die risikoärmer sind. Zudem kann damit erreicht werden, dass mehr Akteure sich die Thematik zu eigen machen, d.h. sich inhaltlich engagieren (was wiederum die Gestaltungsmacht erhöht).

- Begrenzung des Risikos. Hier wird versucht, Entscheidungen reversibel zu machen, die Risiken über Modellversuche abzuschätzen oder, wie aufge-

53 Hood, Ch., The risk game, a.a.O., $21 \mathrm{f}$.

54 Miller, P., Smart swarm, London, 2010, $259 \mathrm{f}$.

55 Die focus group ist eine Methode zur Entwicklung und Prüfung von Ideen sowie zur Kreierung von Konzepten. Der Ansatz wird primär in sozialwissenschaftlichen Phasen der Entwicklung von Forschungskonzepten genutzt, immer öfter aber auch auf praktische Problemlösungsprozesse übertragen (Morgan, D L.: Focus groups, in: Annual Review of Sociology, 22 (1996), 129-152). 
zeigt, ,auf Sicht“ zu fahren. Formal wächst damit zwar die Gestaltungsmacht, aber das kann durchaus auch trügerisch sein: Fehleinschätzungen sind eher wahrscheinlich und das ,auf Sicht“-Fahren begünstigt den status quo.

- Risikoverlagerung. Hierbei bemüht man sich, das Risiko auf bestimmte Regionen oder soziale Gruppen zu konzentrieren, die vermeintlich wenig(er) Widerstand bilden. Das ist eine Strategie, die zwar Gestaltungsmacht suggeriert, aber immer schwerer durchzuhalten ist, weil sie in einer vernetzten und globalisierten Welt eben doch breitere Widerstände auslöst.

\section{(c) Strategie-Selektion}

Bei der Diskussion, welche Strategievariante in der Politik primär verwendet wird, muss zunächst nach dem Problemtypus unterschieden werden. Verteilungsfragen sind anders zu behandeln als neuartige Probleme, deren Definition noch nicht ganz klar ist, geschweige denn, dass man dafür schon Lösungen hätte (sofern die Probleme nicht von Interessengruppen mit dem Ziel besetzt werden, den Staat zu einem bestimmten Handeln zu zwingen und sich damit Verteilungsvorteile $\mathrm{zu}$ verschaffen). Verteilungsprobleme werden traditionell primär hierarchisch zu lösen versucht, sofern es nicht gelingt, damit Tauschgeschäfte zu verbinden. Zunehmend werden aber auch Strategien eingesetzt, die Verteilungsprobleme mit Problemlösungsprozessen zu koppeln oder Verteilungsfragen zu dezentralisieren (Dritter Sektor), indem sich die übergeordnete Politik auf Rahmenvorgaben zurückzieht. Eine besonders elegante Form der Konflikt-Dezentralisierung sind organisierte Wettbewerbsverfahren (etwa bei der Verteilung von Transfermitteln), Verteilungsungleichheit legitimiert sich hierbei über Leistung. Reine Verteilungsprobleme sind allerdings selten, i.d.R. werden sie zu „Problemlösungs-Aufgaben“ umdefiniert, wobei die Verteilungsaufgaben NebenProbleme darstellen sollen.

Problemlösungsprozesse werden dagegen häufiger über netzwerkartige Konsensbildung zwischen sozialen, politischen und fachlichen Eliten bearbeitet - mit allen Problemen, die solche Netzwerke mit sich bringen. Die traditionellen Versuche der Politik, Probleme als ,,technisch“ zu definieren und soziale Implikationen herunterzuspielen, stoßen zunehmend auf Widerstand („Wutbürger") und werden immer mehr in Bürgerbeteiligungsverfahren eingebettet.

Bei der Wahl der Reaktionsformen spielen - neben der Treffsicherheit der Alternative - sowohl die Transaktionskosten (d.h. der politische, teilweise auch ökonomische Aufwand) als auch die Möglichkeiten der Korrektur eine Rolle. Institutionelle Reaktionsmuster setzen einen größeren politischen Kraftaufwand 
voraus und sind deshalb eher mittel- bis längerfristig nutzbar. Dabei wiederum sind institutionelle Änderungen, die Machtstrukturen verändern, mit besonders hohen Transaktionskosten belastet. Sie verlangen entsprechend hohen Aufwand und vollziehen sich langsamer, bedürfen zumeist auch guter „,ideologischer“ Begründung, um Veto-Positionen zu schwächen. Die aktuellen Diskussionen zu Verwaltungsreformen und zur Reform politischer Entscheidungsfindung über Plebiszite fallen darunter. Die empirische Basis (z.B. Kosteneinsparungen bei Verwaltungsreformen, ,fairere“ Bürgerbeteiligung bei Entscheidungen) ist wissenschaftlich schwierig zu belegen, trennt hier freilich die Spreu vom Weizen.

Verwaltungsreformen haben häufig auch einen entscheidungspolitischen Hintergrund: die zentralen Ebenen von Entscheidungsüberlastung zu entlasten und Verteilungsaufgaben möglichst nach unten zu delegieren. Dabei steht nicht im Vordergrund, ob die Entscheidungen dadurch „rationaler“ oder „,kostengünstiger"، werden, sondern ob es gelingt, das politisch-administrative System handlungsfähiger zu machen, d.h. die Tendenzen zur Politik- und Politiker-Verdrossenheit durch stärkere dezentrale Mitwirkungsmöglichkeiten zu reduzieren. Der Erhalt einer den Namen verdienenden Selbstverwaltung und vor allem ehrenamtlicher politischer Betätigung treten hinzu.

\section{Folgerungen}

Was der aufgezeigte Wandel der Politikmodi im Ergebnis für das politische System bedeutet, ist noch schwer abzuschätzen. Einigermaßen verlässlich kann allerdings die Richtung der Veränderung wie folgt gekennzeichnet werden:

Der Wandel der Reaktionsformen geht eindeutig in Richtung eines coping, ${ }^{56}$ d.h. eines reaktiven, sich absichernden Entscheidens. Es beruht in Teilen auf erzwungenem Lernen, in Teilen auf der Notwendigkeit, sich veränderten Rahmenbedingungen anzupassen. Auch spielt eine Rolle, dass sich traditionelle Reaktionsformen, sofern sie als Taktik zu erkennen sind, schnell verschleißen. Die Opposition akzeptiert sie nicht mehr, Medien kritisieren sie oder machen sie lächerlich (vgl. die Wahl von ,,alternativlos“ zum Unwort des Jahres 2010). Andere Gründe sind, dass sich in modernen pluralistischen Demokratien die Zahl der Vetopositionen erheblich erhöht hat, die informatorische Gegenmacht in Verbänden, Medien und der Wissenschaft enorm vergrößerte und es immer mehr Möglichkeiten für die Opposition gibt, sich taktischen Einflüssen zu entziehen. Deshalb kommt dem 
Konsensmanagement, d.h. dem Umgang mit politischen Kosten, eine so große Bedeutung zu.

Vergleicht man die tradierten mit den neueren Reaktionsmustern, so ist festzuhalten, dass letztere sich meist auszeichnen durch: eine verstärkte Kooperation zwischen den Eliten, die an Entscheidungen als Betroffene oder Agierende zu beteiligen sind; eine erweiterte Steuerung durch Zielvorgaben/Rahmensetzungen (parametrische Steuerung) bei wachsenden Spielräumen im konkreten Vollzug; eine Einflussmehrung auf Denkmuster und Einstellungen (paradigmatische Steuerung); sowie schließlich eine Auslagerung von politischen Entscheidungen auf vorbereitende Gremien und Gruppen unterschiedlichster Form. Vor allem ist die Sensibilität der Politik für soziale Fragen deutlich gewachsen: So bekennen sich die Eliten wieder stärker zu ihrer gesellschaftlichen Verantwortung ${ }^{57}$ und wächst die Bereitschaft, die Bürgerschaft relativ früh in Projekt-Entscheidungen einzubinden. Letzteres vor allem dann, wenn das Problemfeld sehr unübersichtlich erscheint, es keine eindeutig richtige Problemlösung gibt und die gewählte Problemlösung hochgradig risikobesetzt ist. In diesem Fall bemüht sich die Politik um „Definitionsgemeinschaften“, die auf eine gemeinsame Problemdefinition, eine für richtig gehaltene Lösung und ein kollektiv gestütztes Verfahren zielen nicht zuletzt, um die Öffentlichkeit auf die gewählte Linie einzuschwören. Das Verfahren ist im Übrigen dem Paradigmenwechsel in den Lehrmeinungen der Sozialwissenschaften nicht unähnlich. ${ }^{58}$

Die Ausrichtung auf eine verstärkte, in Teilen umfassende Kooperation und auf längerfristige Lernprozesse stellt daher die wohl herausragende Veränderung der erkennbaren Politikmodi in Deutschland dar. Ein zentrales Mittel sind in diesem Kontext organisierte Kommunikationsprozesse, ${ }^{59}$ häufig unter professioneller Moderation, im Konfliktfall auch unter Rückgriff auf Mediationsverfahren (die heute bei Großprojekten nahezu üblich geworden sind ${ }^{60}$ ). Immer wichtiger wer-

57 Bunselmeyer, E./Holland-Cunz, M.: Für wen die Verantwortung zählt. Was Deutschlands Entscheidungsträgern wichtig ist und was sie antreibt, in: WZB-Mitteilungen 141/Sept. 2013, 36-39.

58 Ein schönes Beispiel für solche Definitionsgemeinschaften haben Van Assche et al. (Performing success and failure in governance: Dutch planning experiences, in: Public Administration 90(2012), 567-91) für das niederländische Planungssystem aufgezeichnet, das im Ausland ein sehr hohen Ruf genießt, intern aber sehr viel skeptischer gesehen wird.

59 Fürst, D.: Rahmenbedingungen des Einsatzes von Planungsverfahren im öffentlichen Sektor, in: Fisch, R./Beck, D. (Hg.): Komplexitätsmanagement. Methoden zum Umgang mit komplexen Aufgabenstellungen in Wirtschaft, Regierung und Verwaltung, Wiesbaden 2004, $127 \mathrm{f}$.

60 Vgl. Meister, H.-P./Gohl, Ch.: Politische Mediation bei umstrittenen Infrastrukturprojekten - das Beispiel des Frankfurter Flughafens, in: Fisch, R./Beck, D. (Hg.): Komplexitätsmanagement., Wiesbaden 2004, $264 \mathrm{f}$. 
den in diesem Kontext policy communities, mithin Politik- und Akteursnetzwerke, über die sich Politik formiert. Zunehmend scheint sich dahinter der Bedarf zu verbergen, Politikprozesse ggf. zu verlangsamen, um mit deren Komplexität und den sich damit verbindenden Risiken wirksamer umgehen zu können. ${ }^{61}$

Politiker sind in einer offenen Demokratie angesichts wachsamer Medien und unabhängiger Rechtsprechung allerdings nicht frei in der Wahl ihrer Strategien. Zur technischen Qualität tritt die notwendige individuelle wie kollektive Akzeptanz. So können Wähler bekanntlich beträchtliche Veto-Potentiale generieren, direkt gegen Entscheidungen vorgehen oder mit Politikverdrossenheit im Stillen protestieren, falls Politiker lediglich auf Expertengremien ausweichen und damit Verantwortung delegieren - oder auch nur auf Zeitgewinn zielen. ${ }^{62}$ Zwar sind auch in Demokratien Formen der Elitenherrschaft nicht zu vermeiden, weil sich unter Berufspolitikern ,innere Zirkel“ der Macht ausbilden, ${ }^{63}$ die sich wiederum durch „kulturelle Bildung, Milieukenntnisse, habitualisierte Verhaltensmuster und vor allem Netzwerke" von der breiten Masse abheben. ${ }^{64}$ Gleichwohl unterliegen sie immer häufiger kritischer Öffentlichkeit.

In ihren Antrieben hat sich die Politik dagegen kaum nennenswert verändert: Machterhalt steht im Mittelpunkt, und zwar umso mehr, je größer der Anteil der Berufspolitiker in den Parteien ist. Deshalb tendiert Politik dazu, primär auf Druck und die politischen Kosten des Handelns (Konsens- und Risikokosten, eng rückgekoppelt) zu reagieren und solche Lösungen $\mathrm{zu}$ wählen, welche die politischen Kosten niedrig halten. Allerdings ist das Themenfeld um vieles komplexer geworden, weil Gefahrenabwehr (Technikfolgenabschätzung, globale Risiken etc.) immer mehr Aufmerksamkeit absorbiert. Die traditionellen Mechanismen der Politik tendieren gerade dazu, das zu zerstören, was für eine solche Gefahrenabwehr am wichtigsten ist: das Vertrauen in die Politik.

Dieses Vertrauen ist in den vergangenen Jahren geschrumpft. Die Politikmodi bleiben zu wenig auf Vertrauensbildung ausgerichtet. Zudem gewinnt die Öffentlichkeit den Eindruck, dass die Politikinhalte den sich wandelnden Politikmus-

61 Vgl. Korte, K.-R.: Risiko als Regelfall: Über Entscheidungszumutungen in der Politik, in: Zeitschrift für Politikwissenschaft 21(2011), 465-477 (469 f.).

62 Niehaus, E: Die Zerstörung der Parteiendemokratie von oben und unten, in: Aus Politik und Zeitgeschichte 44-45/2011, 11.

63 Die inneren Zirkel werden nach Crouch durch Spitzenpolitiker, Berater und Lobbyisten gebildet, zwischen denen die Individuen hin und her wechseln (Crouch, C: Postdemokratie, Frankfurt/M. 2008, 93f.).

64 Bender, Ch./Wiesendahl, E: „Ehernes Gesetz der Oligarchie“: Ist Demokratie möglich? in: Aus Politik und Zeitgeschichte 44-45/2011, 19. 
tern unterworfen werden: So wird unter dem Eindruck des sog. Neuen Steuerungsmodells im Haushaltswesen auch in „Produkten“ gedacht, weil sie klar definiert und medienwirksam präsentiert werden können. Die Lösungen bleiben aber häufig zu kurzsichtig und vordergründig, schwächen damit eine handwerklich saubere administrative Politikformulierung ${ }^{65}$ und dokumentieren eher die Hoffnung, Schwächen im Entwurf von Problemlösungen im weiteren administrativen Vollzug korrigieren zu können.

So wichtig politische Lernfähigkeit auch ist, sie kollidiert offensichtlich mit dem Bedürfnis nach Planungs- und Handlungssicherheit der Öffentlichkeit. Politiker dürfen in den Augen des Publikums ihre Positionen nicht ohne weiteres aufgrund neuerer Erkenntnisse revidieren, wollen sie nicht als unzuverlässig, Umfaller oder Opportunisten gelten. Veränderte Politikmodi mit Auswirkungen auf politische Inhalte bedürfen deshalb der Erklärung. Folglich wird auch die ,Erklärung von Politik“ im Sinne der Rückgewinnung politischer Definitionsmacht zu einer Aufgabe veränderten politischen Verhaltens. Das wiederum bedeutet: Die Zusammenarbeit von Politik und Medien dürfte im Zuge veränderter Politikmodi noch enger werden. Politikerbefragungen, Talkrunden, Aktivitäten über internetbasierte soziale Netzwerke u.a.m. werden verstärkt in das politische Vertrauensmanagement einbezogen. Dass in diesem Kontext noch Beträchtliches zu leisten ist, macht ein Blick auf die aktuellen innen- wie europapolitischen Herausforderungen deutlich. 